CORRECTION

https://doi.org/10.1038/s41586-019-1045-2

\title{
Author Correction: Spatial and temporal heterogeneity of mouse and human microglia at single-cell resolution
}

Takahiro Masuda, Roman Sankowski, Ori Staszewski, Chotima Böttcher, Lukas Amann, Sagar, Christian Scheiwe, Stefan Nessler, Patrik Kunz, Geert van Loo, Volker Arnd Coenen, Peter Christoph Reinacher, Anna Michel, Ulrich Sure, Ralf Gold, Dominic Grün, Josef Priller, Christine Stadelmann \& Marco Prinz

Correction to: Nature https://doi.org/10.1038/s41586-019-0924-x, published online 13 February 2019.

In this Letter, Dominic Grün and Sagar should have been listed as authors, with the affiliation Max Planck Institute of Immunobiology and Epigenetics (MPI-IE), Freiburg, Germany. These authors established the sequencing platform, supervised the sequencing and helped with bioinformatics (see 'Author contributions'). This study was supported by the DFG (grant GR4980 to S.), the Behrens-WeiseFoundation (to S.), and the Max Planck Society (to S. and D.G.) (see 'Acknowledgements'). The Letter has been corrected online. 